\title{
The Distributed Management Tree - Applying a new Concept for Managing Distributed Applications to E-mail
}

\author{
Vito BAGGIOLINI, Eduardo SOLANA, Jean-Francois PACCINI \\ Mira RAMLUCKUN, Stephane SPAHNI, Jurgens HARMS \\ University of Geneva, SWITZERLAND
}

The "Distributed Management Tree" (DMT) is a hierarchical structure designed for the management of distributed systems. The DMT has the form of an inverted tree, with nodes representing small active units for processing elements of management information. The DMT is not integrated into the system it manages but built next to it, supervising it "from the outside". The DMT has two main functionalities: (1) it extracts and refines information concerning the managed system, and (2) provides a mechanism for specifying and handling actions on the managed system. The nodes are programmed to permanently analyze the information about the managed system and to find out if it is in a normal operational state or not. If a faulty behaviour is detected, the DMT can either fix it autonomously or alert a human administrator, depending on the nature of the error. The different hierarchy levels in the tree represent the information obtained at the terminal nodes views with different levels of detail. Furthermore, they provide means to trigger complex commands and propagate them downwards, decomposing them into more elementary commands. This concept has been applied to the management of E-mail systems. A prototype has been developed for managing an important and heterogeneous fraction of the University's E-mail system. 